\title{
The rich phenomenology of brownian particles in nonlinear potential landscapes
}

\author{
J.M. Sancho ${ }^{1, a}$ and A. M. Lacasta ${ }^{2}$ \\ 1 Departament d'Estructura i Constituents de la Matèria, Universitat de Barcelona, Martí i Franquès \\ 1, E-08028 Barcelona, Spain \\ 2 Departamen de Física Aplicada, Universitat Politèctica de Catalunya, Avda. Doctor Marañón 44, \\ E-08028 Barcelona, Spain
}

\begin{abstract}
Non-interacting brownian particles follows Langevin equations fullfiling fluctuation-dissipation relation between friction and thermal noise. Under a linear potential (constant force) Einstein found a relation between diffusion and transport through mobility. In nonlinear potentials this prediction is only satisfied within the limit of very large constant external forces. Moreover, other more interesting behaviors do appear, such as: dispersionless transport, sorting, giant diffusion, subdiffusion, superdiffusion, subtransport, etc. All these phenomena depend on the characteristics of the nonlinear potential landscape: periodic or random, the symmetries and boundary conditions. Moreover, the presence of transport is the keystone of most of this phenomenology. In this review we present numerical simulations of these facts and theoretical analysis when possible.
\end{abstract}

\section{Introduction}

The motion of non-interacting brownian particles, studied by Einstein a century ago[?], is still attracting interest for its relevance in understanding the relation between transport and diffusion processes. Nevertheless, not all the phenomenology is so simple, as can be inferred from Einstein's solution of the problem. Actually, Einstein's relation connecting the diffusion constant and mobility is not fulfilled when particles move in a nonlinear potential landscape [2]. Furthermore, the absence of particle interaction discards some very appealing cooperative effects, but as we show here, a very rich new phenomenology emerge if nonlinearities and thermal fluctuations cooperate. In the most simple situation, these brownian particles obey Newton's equation with Stokes friction and thermal stochastic force. The nonlinearities enter through an external potential. The rich phenomenology needs neither the sophisticated nongaussian noises nor the fractional Fokker-Planck equations. The interest for such a simple system has increased due to its experimental relevance in the motion of dilute colloidal particles on modulated surfaces [3-7].

This simplest scenario starts with the Newtonian equation. It consists of non-interacting particles moving on a two-dimensional potential, subjected to an external force and also to thermal noise and the associated dissipation. The equations of motion are:

$$
\begin{aligned}
& m \ddot{x}=-\frac{\partial}{\partial x} V(x / \lambda, y / \lambda)-\eta \dot{x}+f_{x}+\xi_{x}(t) \\
& m \ddot{y}=-\frac{\partial}{\partial y} V(x / \lambda, y / \lambda)-\eta \dot{y}+f_{y}+\xi_{y}(t) .
\end{aligned}
$$

\footnotetext{
a e-mail: jmsancho@ecm.ub.es
} 
The modulation of the surface is described by a potential $V(x, y)$ of height or depth $V_{0}$ and spatial scale $\lambda$, which for simplicity we take to be equal in the $x$ and $y$ directions. A constant external force with Cartesian components $f_{x}, f_{y}$ (magnitude $f$ ) acts on the particle. The Stokes coefficient of friction is $\eta$, and the thermal environment is captured by the mutually uncorrelated white noises $\xi_{i}(t)$ that obey the fluctuation-dissipation relation at temperature $\mathrm{T}$,

$$
\left\langle\xi_{i}(t) \xi_{j}\left(t^{\prime}\right)\right\rangle=2 \mu k_{B} \mathrm{~T} \delta_{i j} \delta\left(t-t^{\prime}\right) .
$$

In order to simplify the excess of parameters, we make the following changes: $r_{x}=x / \lambda, r_{y}=y / \lambda$ and $\tau=\left(V_{0} / m\right)^{1 / 2} t / \lambda$. The three independent parameters are then the effective friction, force and temperature, respectively,

$$
\gamma=\frac{\eta \lambda}{\sqrt{m V_{0}}}, \quad F=\frac{\lambda f}{V_{0}}, \quad T=\frac{k_{B} \mathrm{~T}}{V_{0}} .
$$

In many experimental situations (low Reynold's number) the motion is overdamped $(m=0)$, and the temporal change is different $\tau=V_{0} t / \eta \lambda^{2}$, but now the two independent parameters $F$ and $T$ are the same as in (3).

The potential can be periodic, partially random or totally random, but always yielding nonlinear forces. It is worth remarking here the relevance of the interplay between the potential $V\left(r_{x}, r_{y}\right)$, the constant external force $F$ and the temperature $T$ for understanding the rich phenomenology.

In the presence of a constant force $F$, particles are transported forward and exhibit a global finite mean velocity,

$$
\langle v\rangle=\lim _{\tau \rightarrow \infty} \frac{\langle r(\tau)\rangle}{\tau},
$$

where $\langle\ldots\rangle$ means statistical average over the realizations of the noise $\xi(\tau)$ and initial conditions. From the above equation one can define the mobility coefficient $\mu$ associated to transport as,

$$
\mu=\frac{d\langle v\rangle}{d F}
$$

Particles move forward, but they also disperse, and one can characterize this motion by the diffusion coefficient $D$,

$$
D=\lim _{\tau \rightarrow \infty} \frac{\left\langle r(\tau)^{2}\right\rangle-\langle r(\tau)\rangle^{2}}{2 \tau}
$$

A warning is in order here. Theoretically one should take the limit $\tau \rightarrow \infty$, but in practical situations the total observation time interval is finite, although it should be much larger than any other characteristic time of the system.

In the Einstein analysis of the motion of non interacting Brownian particles in flat potentials we have that $\langle v\rangle=F / \gamma$, and $D=T / \gamma$, which gives the famous Einstein relation between diffusion and transport,

$$
D=T \mu=T \frac{d\langle v\rangle}{d F} .
$$

This expression is used as a reference in our analysis of transport and diffusion. We will see that it does not hold if the external potential is nonlinear, except for very large values of $F$. This is not the principal anomaly in this study. We discuss here a rich phenomenology of transport and diffusion, such as:

- Dispersionless regime: For some interval of forces, there is a transient regime exhibiting null diffusion [8].

- Giant diffusion: The diffusion coefficient grows several orders of magnitude in a particular domain of the force $F[9-13]$.

- Sorting: In two dimensions and a periodic potential, an orthogonal component of the mean velocity appears with respect to the driving force $F[3-7,9,14-17]$. 
- Subdiffusion and superdiffusion: In the case of random potentials, we find subdiffusive and superdiffusive regimes, but with finite transport $[18,19]$.

- Subtransport: As in the previous case, and for some values of the force, a subtransport regime is found [20].

This rich phenomenology is associated with the following facts, which are very easy to check. The anomalies in transport are related with the behavior of mobility, which presents a pronounced maximum for some critical value of the force. The anomalies in the diffusion (dispersionless) are either associated with the mentioned anomaly or with the randomness of the scape times from the potential wells. The sorting phenomenon are related with the geometry of the system and with the laminar flow associated with the force. It is worth remarking that when transport is not present, the phenomenology is drastically reduced. Actually, the cooperation between transport and diffusion exhibits a greater variety of phenomena.

Finally, we remark that this approach based on classical Langevin equations can also be applied to the most simple case of particle interaction: a dimer molecule. The interaction is only present in a pair of particles connected by an harmonic spring. Either diffusion or transport is currently studied: diffusion [21,22], transport and diffusion [23,24], bistability and hysteresis [25], and dimers in a ratchet scenario [26]. A futher example is the hard core particle-particle interaction with elastic collisions between neighboring particles [27].

It is our objective here to address systematically the correlations between transport and diffusion phenomena of noninteracting brownian particles in tilted periodic potentials, exploring the relevance of the main parameters, such as: temperature, external force and friction. Our study is mostly numerical by simulations of the stochastic Langevin equations of an ensemble of independent particles, and by performing the corresponding statistical averages to get $\langle r(\tau)\rangle$ and $\left\langle r(\tau)^{2}\right\rangle$. It is complemented with theoretical analysis.

This minireview is organized as follows: In the next two sections we address the case of periodic potentials. In Section 2, we discuss the behavior of Einstein's relations for a periodic potential, the giant diffusion and dispersionless regime. In Section 3, we analyse the sorting phenomenon and the relevance of the geometry. In Section 4, we study the anomalies in transport and diffusion due to random potentials. Finally, we end with some conclusions and perspectives.

\section{Transport and diffusion in periodic potentials}

Our starting point is the Langevin equation (1) with the periodic potentials

$$
V(r)=\frac{V_{0}}{2} \cos (2 \pi r), \quad V\left(r_{x}, r_{y}\right)=\frac{V_{0}}{2}\left[\cos \left(2 \pi r_{x}\right)+\cos \left(2 \pi r_{y}\right)\right],
$$

for one or two dimensions, respectively. The two potentials have a barrier of height $V_{0}$ connecting neighbouring wells. This choice makes the comparison between the results of both cases easier. Particles perceive the wells of this potential and the tilted force $F$. In the adimensional variables and for the critical value $F_{c}=\pi$, the system presents a saddle point (Fig. 1-left). For small forces, particles remain at the minima during large sojourn times with zero velocity: this is called the "locked" state. For large forces the potential landscape has no potential wells, and the mean velocity saturates to the free particle value $v_{0}=F / \gamma$, which is called the "running" state. For some intermediate force values, these two states combine. This regime can be characterized by a critical force which does depend on the friction parameter. In this region, the interplay between locked and running states gives the most interesting phenomenology.

An exhaustive analytical study of this system was that by Risken [28] where mobilities, distribution functions, eingenvalues, etc., were obtained. Nevertheless, little emphasis was placed on observational phenomenology associates with explicit trajectories and transient anomalies. Moreover, the literature was focused on one-dimensional overdamped situations, overlooking the new phenomenology which appears when friction is very small. 

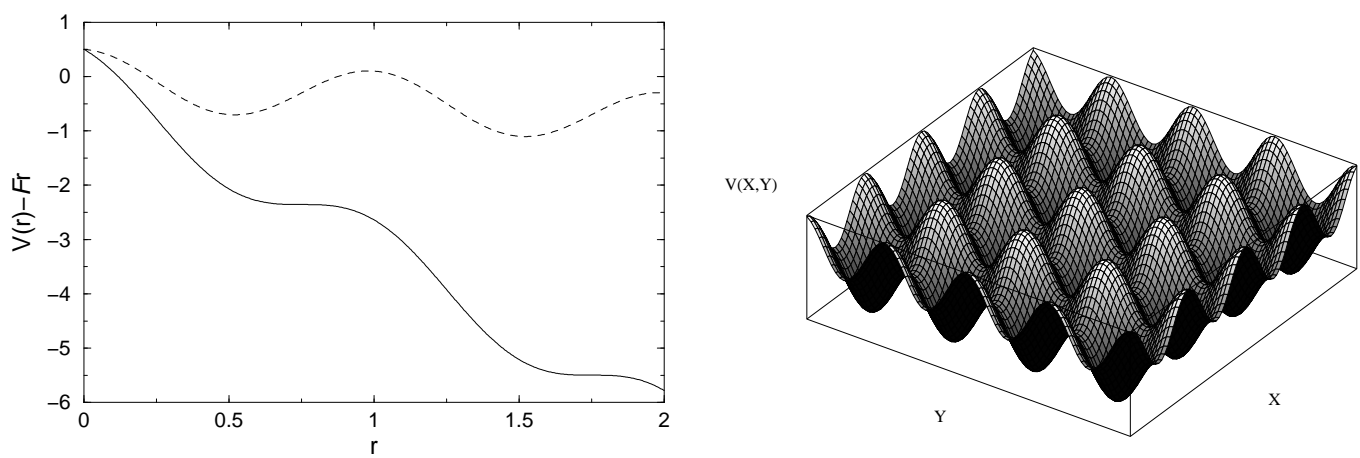

Fig. 1. Left: Tilted one dimensional periodic potential for two forces: $F<F_{c}$ (broken line) and $F \sim F_{c}$ (continuous line), where $F_{c}=\pi$ is the deterministic critical force. In the first case maxima and minima are clearly seen. In the second case we have saddle points. Right: two dimensional periodic potential.
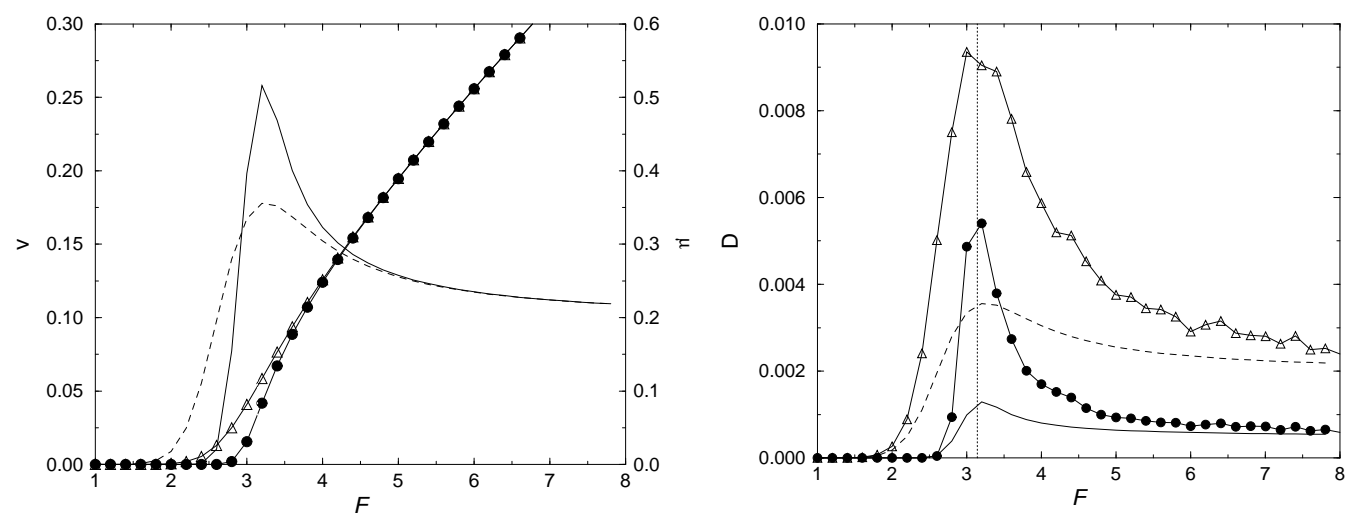

Fig. 2. Left panel: Average velocity (symbols, left y-axis) and mobility (lines, right y-axis) versus force for two noise intensities $T=0.01$ (circles and continuous line) and $T=0.04$ (triangles and dashed line). Friction is $\gamma=20$. Right panel: Diffusion (symbols) and Einstein relation (lines) for the same parameters as in left panel.

\subsection{Overdamped regime}

The first situation in our study is the overdamped limit of (1), which has been extensively studied in the literature. When there is no force, we do not have net transport, and diffusion is due to the particle jumping from one well to a neighboring one. This is controlled by the Kramers barrier crossing mechanism and no surprising phenomenology is expected. Here we focus on the most relevant aspects involving transport. In Fig. 2, we present numerical results for velocity, mobility and diffusion in this regime. In the left panel we see that for small forces the velocity is exponentially small, but after the critical value $F_{c}$ the velocity recovers the free value, as expected. Moreover, mobility has its maximum near $F_{c}$. In the right panel, we plot the diffusion and the Einstein relation (7). It is clear from the figure that the Einstein relation is only fulfilled in the very large force limit. Thus there is no simple proportionality relation between diffusion and mobility [29].

The enhancement of the diffusion near the critical force can be quantified by using a scaling representation. Low temperatures magnify this fact [11-13]. An analytical calculation of this behavior predicts the following scaling relation $[9,10]$,

$$
\frac{D}{T^{1 / 3}} \sim \phi\left(\left(F-F_{c}\right) T^{-2 / 3}\right)
$$


where $\phi$ is the scaling function $[10,12]$. In Fig. 3 , we see what this scaling looks like.

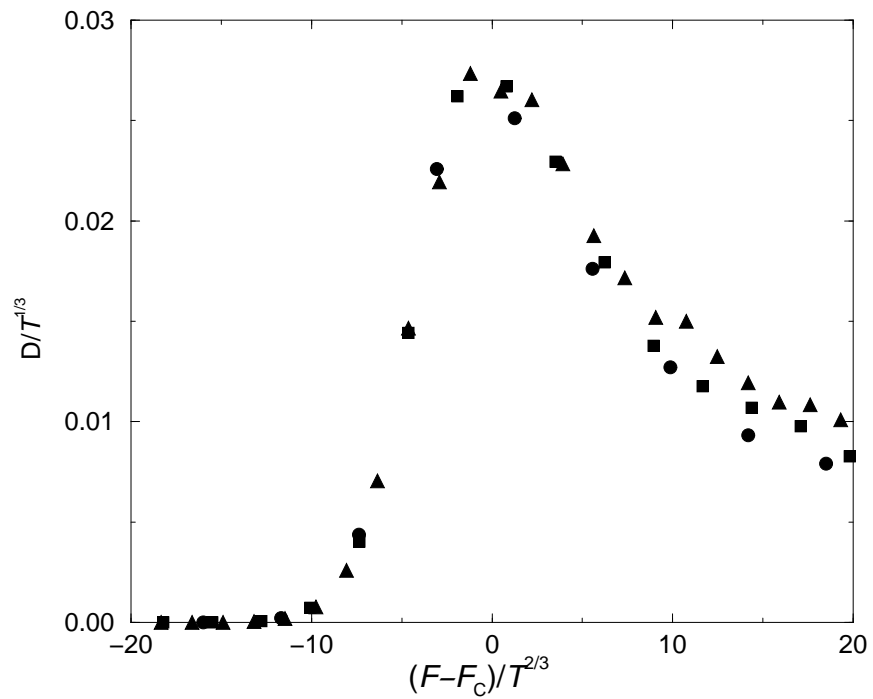

Fig. 3. Scaled diffusion in the large friction regime for various temperatures: $T=0.01$ (circles), 0.02 (squares) and 0.04 (triangles), according to the theoretical prediction (9).

Although this enhancement is manifest in the critical force domain (Fig. 2), it is far from being a giant $[12,13]$. In fact, diffusion decreases as $D \sim T^{1 / 3}$ in absolute values. If we divide this result by the free-particle diffusion value $D_{0} \sim T$, we get that $D / D_{0} \sim T^{-2 / 3}$, which actually grows when $T$ decreases, but this is an artifact of the definition.

\subsection{Infradamped regime}

Now we will see the relevance of a very small friction which cooperates with transport and diffusion. The new phenomenology emerging in this case has received little attention in the literature. Extensive numerical simulation data reveal different types of anomalies. Before entering into discussion of these phenomena, we present the diffusion behavior without transport $F=0$.

The variance evolution for four different friction values is showed in Fig. 4. We see that after some ballistic transient, the linear growth is recovered, but with larger diffusion for smaller friction. Much more interesting is the behavior of single particle trajectories. In the large friction regime, we observe thermal fluctuations inside the wells with random jumps between neighboring wells. Nevertheless, in the very small friction case we see large jumps emulating Lévy flights (Fig. 5). The dependence of diffusion versus friction in a wide domain range is presented in the same figure. Analytical calculations of the diffusion $[18,19]$ predict the following asymptotic expressions,

$$
D \sim \frac{\pi}{\gamma} e^{-1 / T}, \quad D \sim \frac{\pi T}{4 \gamma} e^{-1 / T} .
$$

These expressions correspond to the large or small friction limits, and their corresponding predictions are compared with numerical simulation in Fig. 5.

The next step is to include the force in order to observe the qualitative changes with respect to the previous scenario. We present the numerical data (Fig. 6) of the velocity and mobility versus force for three different values of the friction. By defining the critical force at the point where mobility has a maximum, we can see that the critical force now depends on the friction 


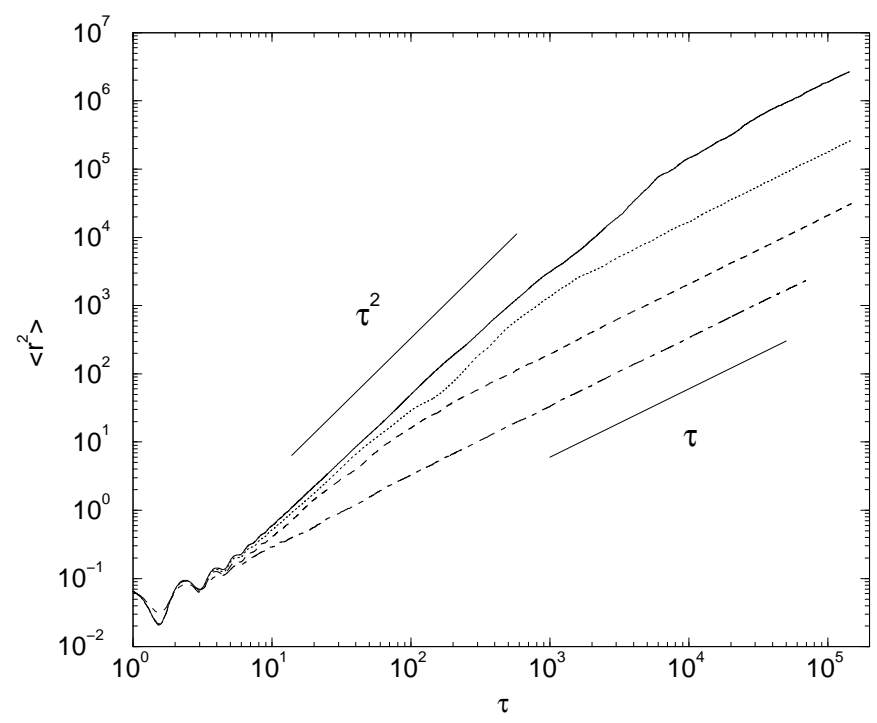

Fig. 4. Second moment evolution, corresponding to the periodic potential, for $\gamma=0.0001$ (solid line), 0.001 (dotted line), 0.01 (dashed line) and 0.1 (dot-dashed line) $(F=0, T=0.2)$.

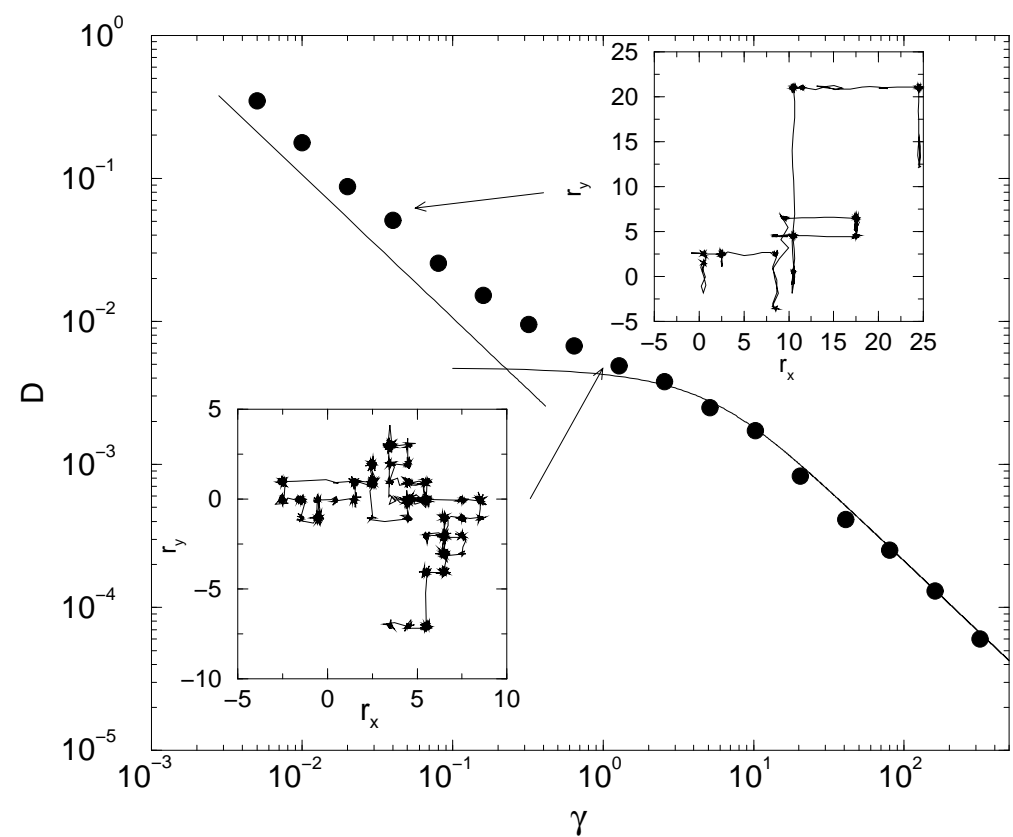

Fig. 5. Diffusion versus friction. Lines correspond to the theoretical predictions (10). Insets: single particle trajectories in the high friction (left) and low friction (right) regimes $(T=0)$.

as it becomes smaller for smaller frictions. This phenomenom is curious, and its analytical explanation is given in [28].

Moreover, a more unexpected phenomenology appears in the dispersion in a wide domain of forces larger than the critical force. In Fig. 7 (left) we see that the evolution of the variance exhibit flat regimes where diffusion is zero. This is quite an interesting anomaly, because noise is not too small and force is also finite. Moreover, the mean velocity and the mobility are well 


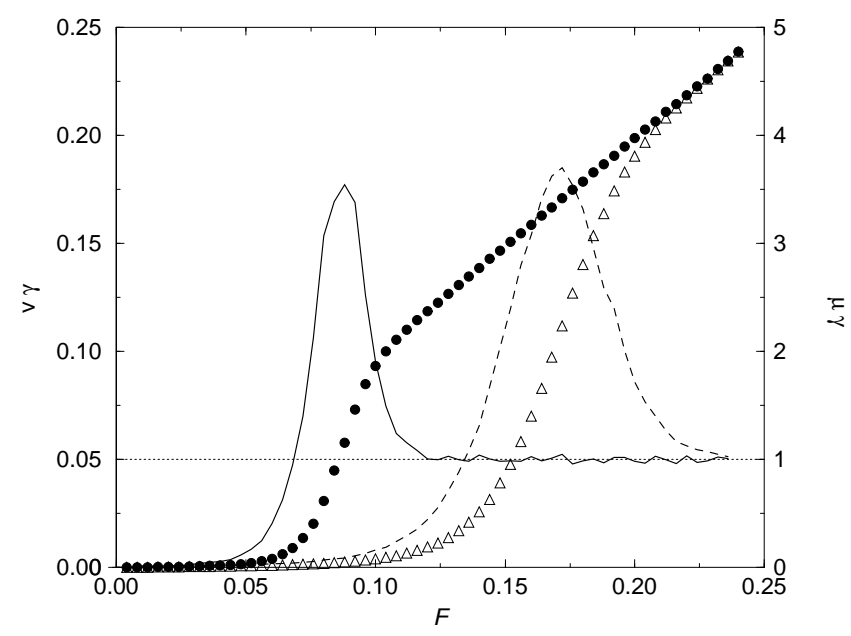

Fig. 6. Velocity (left y-axis) and mobility (right y-axis) versus force for two friction values: $\gamma=0.04$ (circles and solid line) and 0.08 (triangles and dashed line) $(T=0.2)$. The maxima of the mobility determines the critical force which in these cases depends on the friction.
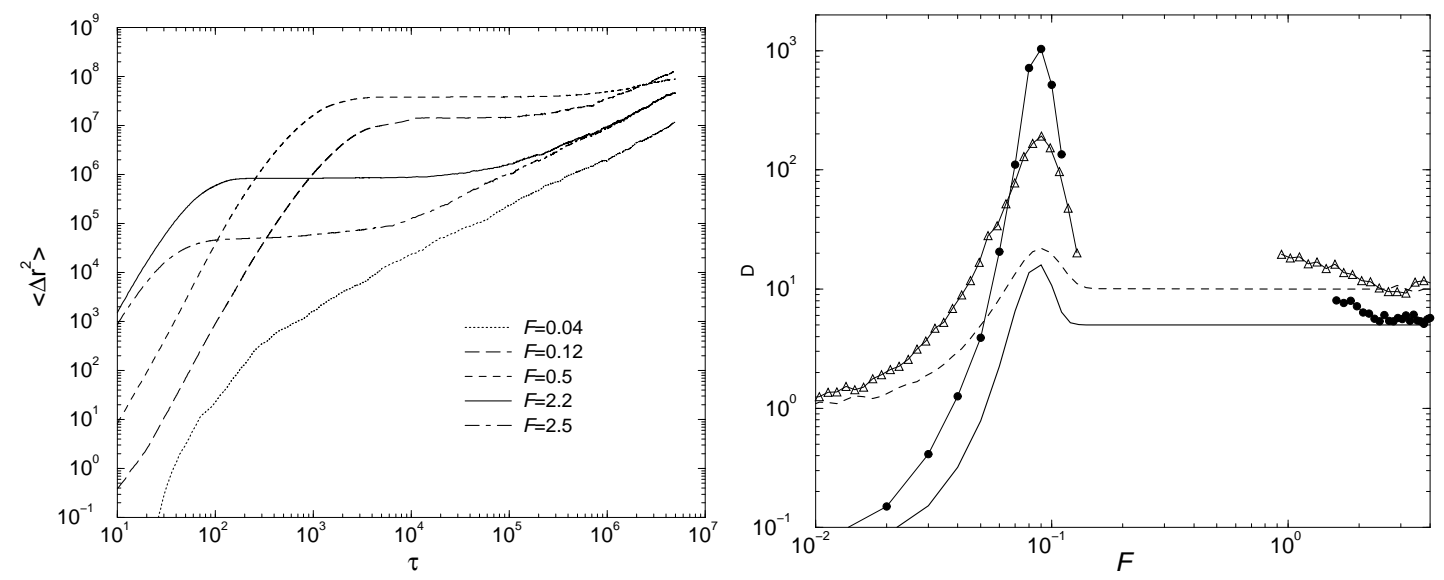

Fig. 7. (Left) Time evolution of the variance versus time for different forces. The domain with horizontal trajectory is the dispersionless regime. (Right) Simulation results of the diffusion coefficient versus forces (symbols). The empty domain indicates that the steady state was not achieve at the observation time $\sim 10^{7}$. Parameter values: T: 0.2 (circles), 0.4 (triangles). Lower lines correspond to the Einstein relation respectively.

defined, as can be seen in Fig. 6. The physical mechanism underlining this behavior is very simple [8]. Initially, all the particles start in one potential well. They escape according to an exponential distribution corresponding to the probability of exit times. When particles merge, they move with a constant velocity $\sim F / \gamma$. The temporal distribution can thus be transformed into a moving spatial distribution which does not change very much in time. The consequence is that the variance of the position remains constant and diffusion is zero.

Furthermore, diffusion now presents more interesting behaviors. It cannot be evaluated numerically in a region of intermediate forces larger than the critical one (7). We also see, at the critical force, a very giant diffusion of more than two orders of magnitude in absolute values with respect to Einstein's relation. The diffusion becomes larger at smaller values of the temperature. This behaviour is the contrary to that of the overdamped regime. In the limit of large forces, we recover the Eisteins relation, as expected. 


\section{Sorting of particles driven through periodic potential landscapes}

Sorting of colloidal particles of different sizes is a very interesting phenomenon due to its appealing perspectives in applied physics. It is possible to control and target the direct transport and delivery of substances such as DNA fragments or functionalized colloidal particles in biology, materials science, and other technological contexts. A successful methodology involves the flow of a mixture of particles over designed surfaces presenting a periodic array of traps (optical tweezers)[4,5] or microfabricated obstacles [6]. The trajectories of the particles over these surfaces deviate from the direction of flow as the particles are attracted (traps) or repelled (obstacles) by the features of the landscape. The deviation of the particles from that of the flow depends on particle size (or on some other particle characteristic), and hence the particles can be sorted according to trajectory direction. In our study, we use the two-dimensional equations of motion (1). In addition to lattice geometry and potential function parameters, the problem is defined by four independent (dimensionless scaled) parameters: the temperature $T$, the dissipation $\gamma$, the magnitude $F$ of the external force (see Eq.3) and the angle $\theta$ between the external force and the $x$ axis $\left(\tan \theta=F_{y} / F_{x}\right)$.

The potential is chosen to exhibit the main features of the experimental surfaces:

$$
V\left(r_{x}, r_{y}\right)=\frac{ \pm V_{0}}{1+e^{-g\left(r_{x}, r_{y}\right)}},
$$

where $g\left(r_{x}, r_{y}\right)$ is the periodic two-dimensional function

$$
g\left(r_{x}, r_{y}\right)=A\left[\cos \left(2 \pi r_{x}\right)+\cos \left(2 \pi r_{y}\right)-2 B\right] .
$$

and the signs + and - stand for wells and obstacles, respectively. $A$ controls the steepness of these features, and $B$ determines the relative size of a well or obstacle with respect to the spatial period $\lambda$. Here we set $A=5$ and analyze the effect of parameter $B$. In this paper, results for the case of obstacles are presented. Arrangements of traps or mixed traps and obstacles lead to essentially the same phenomenology (see Refs. [14-16]). An example of arrangement of obstacles is illustrated in the left panel of Fig. 8.

Although in all of our simulations we implement the full equations of motion, it should be noted that in the regime of interest in the sorting problem, two simplifications are appropriate: The first is that the dissipation parameter $\gamma$ is large, i.e., the system is overdamped, so that the second derivative term can be dropped entirely. This is of great help in any analytical work [17]. In all of our simulations, we set $\gamma=20$. The second is that thermal effects are unimportant. However, by retaining thermal fluctuations in our simulations, we are able to show the extent to which these fluctuations affect the results. In the right panel of Fig. 8. some typical particle trajectories for different angles $\theta$ are shown.

The sorting capability can be analayzed by studying the mean particle velocity as a function of the relevant parameters. For any given potential, the components parallel $\left\langle v_{\|}\right\rangle$and perpendicular $\left\langle v_{\perp}\right\rangle$ to the force $F$ are constructed from the Cartesian components of the average velocity $\left\langle v_{x}\right\rangle$ and $\left\langle v_{y}\right\rangle$. The magnitude of the deviation of the direction of the average particle velocity from that of the force can be characterized by either of the angles $\alpha$ (called the deflection angle) and $\psi=\alpha+\theta$ (called the velocity angle), defined as

$$
\tan \alpha=\frac{\left\langle v_{\perp}\right\rangle}{\left\langle v_{\|}\right\rangle}, \quad \tan \psi=\frac{\left\langle v_{y}\right\rangle}{\left\langle v_{x}\right\rangle} .
$$

In Fig.9 the angles $\alpha$ and $\psi$ are plotted against the force direction $\theta$. We observe a pronounced series of plateaus along which $\Psi$ is independent of $\theta$ (left panel). These plateaus, which are also observed for traps and mixed arrangements [14-16], can be explained with simple geometrical calculations[16]. The deflection angle $\theta$ (right panel) shows that sorting is possible, because for a given force direction and magnitude, it depends on the size of the particles (parameter $B$ ). For a pair of particle sizes, one can choose $\theta$ so as to optimize the sorting of the particles. For example, the sorting of particles corresponding to $B=0.5$ (solid) and $B=0.7$ 

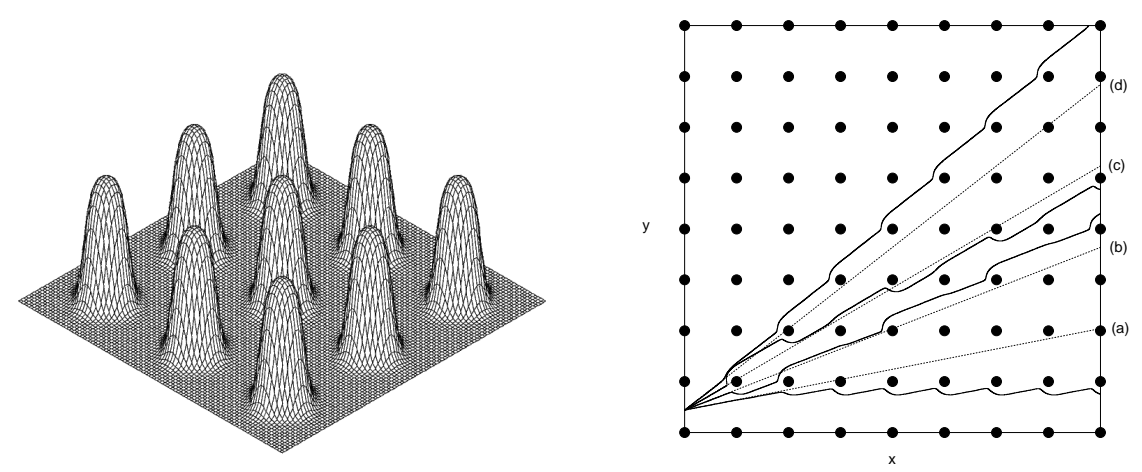

Fig. 8. Left panel: a finite portion of a two-dimensional potential with periodically located obstacles connected by flat plateaus. Right panel: typical particle trajectories for forces applied at different angles represented by the dotted lines. Parameter values are: $B=0.7, \mathcal{T}=10^{-4}$ and $F=8$.
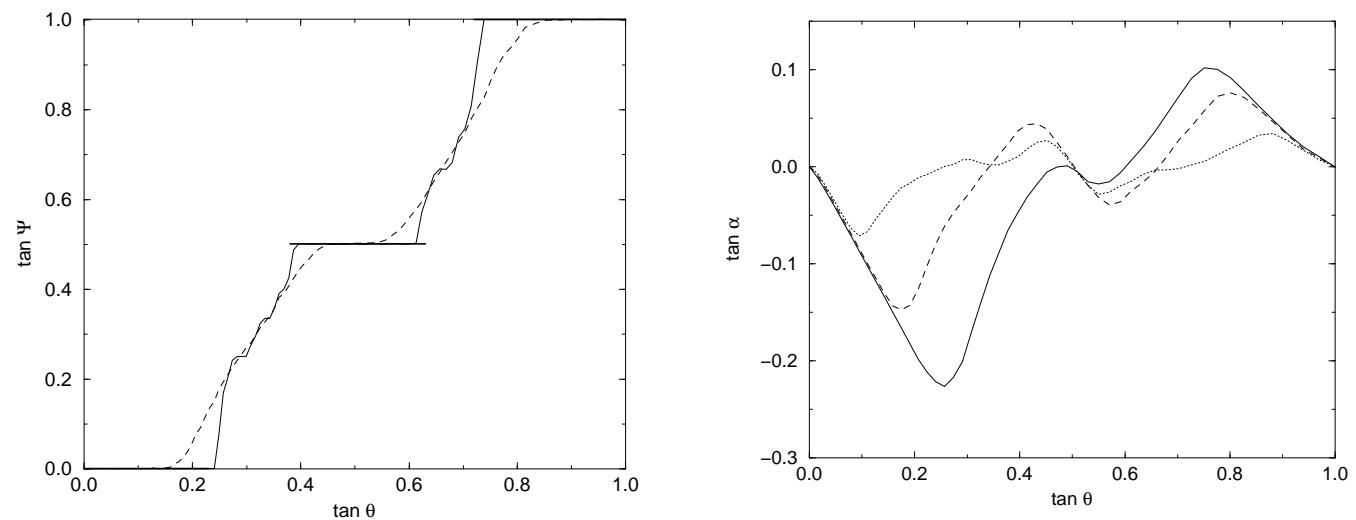

Fig. 9. Plateaus in the absolute velocity angle (left panel) and deflection angle (right panel) for a potential with $B=0.5$ (solid), 0.7 (dashed), and 0.9 (dotted). Other parameters are: $F=10$ and $T=10^{-4}$.

(dashed) is optimized at a force angle $\tan \theta \sim 0.24$, while $B=0.7$ and $B=0.9$ (dotted) are most effectively sorted if one chooses $\tan \theta \sim 0.15$.

In the left panel of Fig. 10 we show the deviation angle as a function of the particle size parameter $B$ for $\tan \theta=0.24$ and three different forces. In all the cases a greater deviation angle for a given particle size is observed for lower forces. It is clear that there must be a range of forces that is most useful for sorting. If the external force is too weak, the particles will become trapped in potential wells or will not be able to get around obstacles. If the force is too strong, the particles will essentially ignore the traps or obstacles and will simply follow the force. The range of forces for successful sorting lies between these two extremes, and for appropriately chosen force directions it is in fact possible to select an optimal force to achieve most efficient separation. This is shown in the right panel of Fig. 10. On the vertical axes, $\beta$ stands for either the deviation angle $\alpha$ for particles of a given size, or for the difference in deviation angles for particles of two sizes. This difference, which one seeks to optimize, is related to a commonly used "figure of merit" for sorting (see Fig. 3 of Ref. [3]).

While the difference in deflection angles is a crucial measure of the sorting capability of a modulated surface, one should also take into account the dispersion of the particles as they reach the end of the system. If this dispersion is too large, e.g., if the particle distribution were to be too broad around the deflection angle so that the distributions of different mean deflection angles overlap, then a measure based only on these averages may not be sufficient 

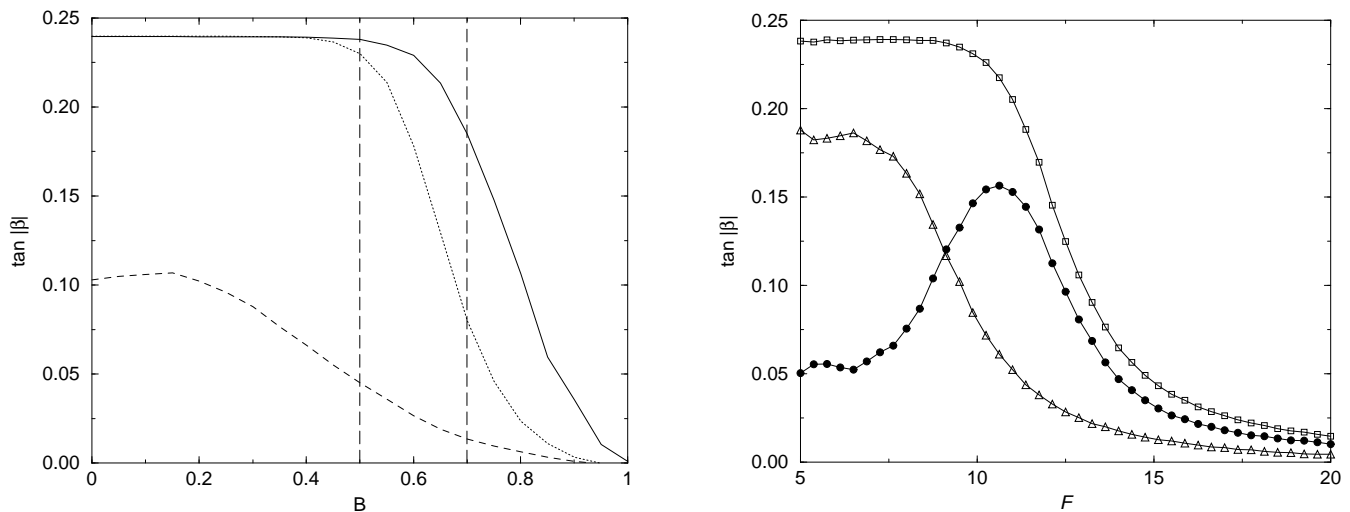

Fig. 10. Left panel: deflection angle vs particle size parameter $B$ for forces $F=5$ (solid), $F=10$ (dotted) and $F=15$ (dashed). Right panel: deflection angle vs $F$ for $B=0.5$ (squares) and $B=0.7$ (triangles). The circles show the difference between the two deflection angles. In both panels the force direction is $\tan \theta=0.24$.
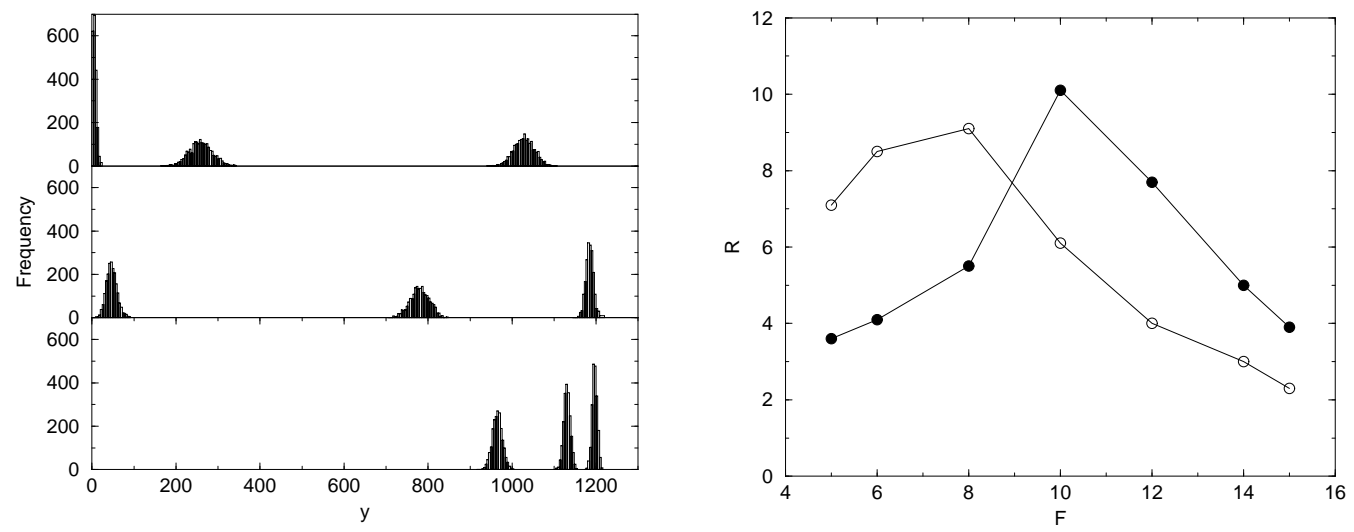

Fig. 11. Left panel: $y$-position arrival distributions for three sets of 2000 particles collected at $L_{x}=$ 5000: $B=0.5,0.7$, and 0.9 (left to right). Upper: $F=5$. Middle: $F=10$. Lower: $F=15$. Right panel: Resolution as a function of the magnitude of the external force for particles of two different sizes: $B=0.5$ and 0.7 (solid circles), $B=0.7$ and 0.9 (open circles). In both panels the force direction is $\tan \theta=0.24$.

for a good selection process. In the left panel of Fig. 11 we show the distributions associated with the deflection angle results in Fig. 10.

Given a set of particles of two different sizes, the resolution $R$ is calculated from the $y$ position distributions for the two types of particles (collected at a fixed $x$-position) as

$$
R=\frac{\Delta Y}{2 \sigma_{1}+2 \sigma_{2}}
$$

where $\Delta Y$ is the peak separation and $2 \sigma_{i}$ are the bandwidths. Resolvable distributions are associated with values of $R \sim 1$ [30]. The resolution for the deflection angles and associated distributions shown in Figs. 10 and 11-left are shown in right panel of Fig. 11. The maxima in the resolution curves indicate the optimal force for sorting of particles of those particular sizes and force directions.

Analytical predictions for the deflection angle have been obtained in Ref. [17]. As based on perturbation expansions, they are only valid for large external forces and/or high temperatures. It would be desirable to develope analytical approaches beyond the perturbative ones. 

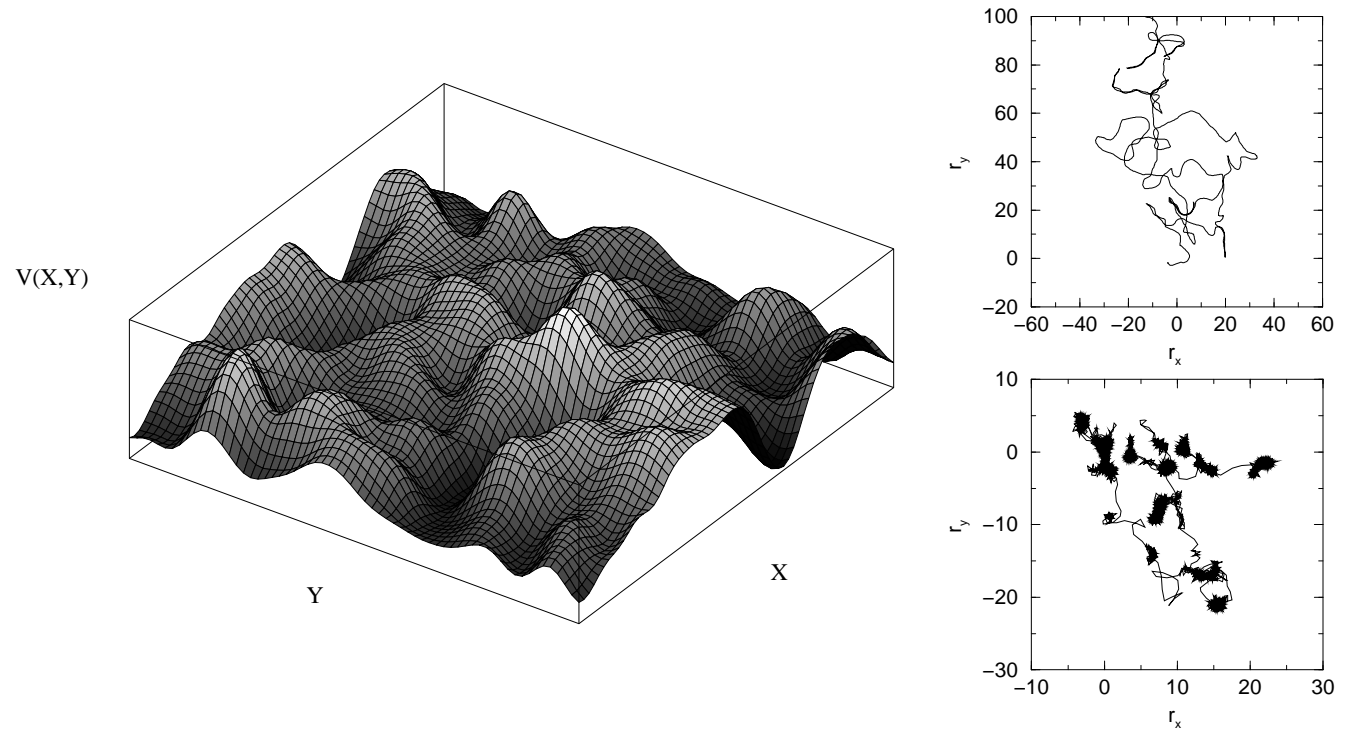

Fig. 12. Left panel: Random potential landscape corresponding to correlation (15) for the parameter values: $V_{0}=1.4, \lambda=4$. Right panel: Particle trajectories in the low friction $\gamma=0.001$ (top) or high friction $\gamma=0.2$ (bottom) cases $(T=0.2)$.

\section{Random potentials: anomalous transport, subdiffusion and superdiffusion}

Random potentials have recently received attention because they can represent realistic spatial disorder $[31,32]$. In this case the relevant parameters are: the intensity $V_{0}$, characteristic length $\lambda$ and the statistical properties such as the spatial correlation $C(x / \lambda)$. If we assume gaussian disorder of zero mean value, we only need the correlation function to make a good characterization of the desired potential,

$$
\left\langle V(x) V\left(x^{\prime}\right)\right\rangle=C(x / \lambda) .
$$

A widely correlation used by our group is the Gaussian distribution,

$$
C(x / \lambda)=\frac{V_{0}^{2}}{2} e^{-\frac{x^{2}}{2 \lambda^{2}}}
$$

which can easily be implemented [33]. In the case of a two dimensional space, the generalization is straightforward and an illustration is presented in Fig. 12.

Random potentials also exhibit the sorting property of periodic potentials, although the effect is quantitatively small. Analytical approximate calculations can be performed in some parameter regimes for transport [17] and diffusion [34]. Nevertheless, no analytical result is available for the most interesting regimes in the domains of small temperature and intermediate forces. In order to see new phenomena, we need to explore these domains by the use of numerical simulations.

\subsection{Diffusion}

Diffusion of overdamped particles in random potentials in one and two dimensions were studied numerically in a preliminary paper [35]. The main result was that in the overdamped regime the motion of the particles are subdiffusive with a nonclassical exponent which depends on the potential characteristics, temperature and spatial dimension. By defining the anomalous exponent $\alpha$ as,

$$
\left\langle\Delta r(\tau)^{2}\right\rangle=A+B \tau^{\alpha},
$$


one can get the exponent by a fit of this expression with the numerical results. Using renormalization group calculations [36], one can fit the numerically found exponents to the following linear dependence

$$
\alpha^{-1}=a+b \frac{C(0)}{D_{0}^{2}} \geq 1
$$

where $C(0)$ takes into account the effects of the random potential parameters such its intensity and spatial scale. $D_{0}=T / \gamma$ is the diffusion of free particles which incorporates the effect of the thermal fluctuations. For all values of the parameters, a subdiffusive regime is found.

When the effect of the friction is studied, one can observe in the numerical data that for lower values of the friction we go from subdiffusion to superdiffusion $[18,19]$. This is a remarkable result although it can be a long transient phenomena because numerical simulations are performed during a finite time and in a finite system. In any case the superdiffusive behavior is clearly seen in the simulations. In Fig. 13 we see the diffusion evolution where superdiffusive,

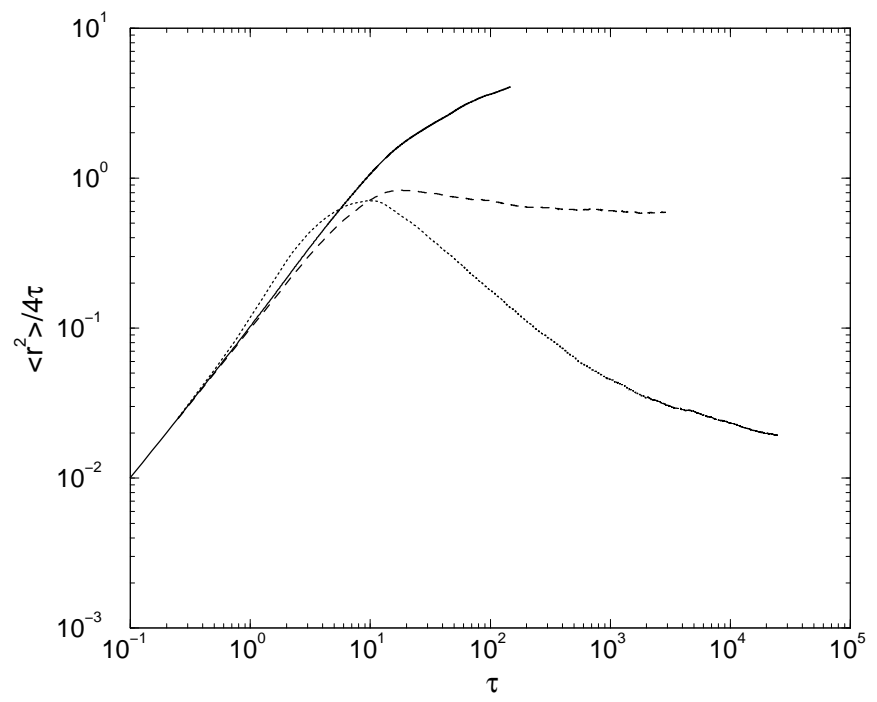

Fig. 13. Dynamical evolution of the diffusion coefficient for a $2 D$-random potential for $V_{0}=0.24$, $\gamma=10^{-4}$ (solid line), $V_{0}=0.24, \gamma=0.1$ (dashed line) and $V_{0}=0.70, \gamma=0.1$ (dotted line). $(F=0$, $T=0.2)$.

normal and subdiffusive regimes are manifested [37].

\subsection{Transport and diffusion}

The phenomenology is richer in the presence of a force because the transport makes qualitative changes in the diffusion. Here we present very preliminary numerical results for the motion in a one dimensional space and in the overdamped regime.

In Fig. 14 we plot the mean velocity (left) and effective diffusion coefficient (right) for four forces obtained from a multiple average (particles and potentials) of the stochastic trajectories . In the left figure we see two very different regimes in transport. For very small forces we observe a subtransport regime, but for larger forces the normal transport regime appears. This anomaly is stable during the time of the observation. The diffusion presents more regimes (right plot). We see here the evolution of the diffusion, and three regimes are appreciated: normal diffusion for large forces, superdiffusion for intermediate forces and subdiffusion for small forces. Simulation of the anomalous regimes lasts long time when compared with the deterministic characteristic 

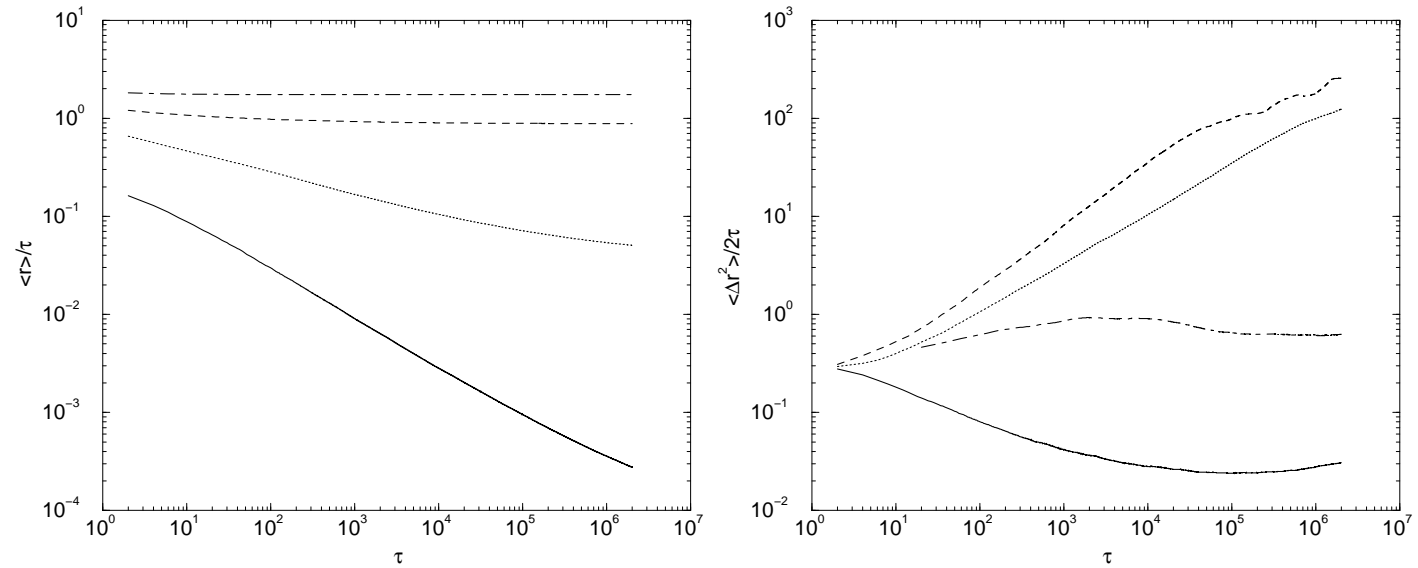

Fig. 14. One dimensional simulation results of transport and diffusion in a random potential for four forces. (Left) Mean velocity versus time. (Right) Diffusion versus time. Force values: 0.2 (solid), 0.9 (dotted), 1.4 (dashed) and 2.0 (dot-dashed). Other parameters: $V_{0}=1, \lambda=1, T=0.2$.

time, $t_{0}=\lambda / v_{0}=1 / F$. More massive simulations are necessary for a better characterization of these regimes. Moreover, in the case of random potentials no analytical results are available yet. Further work is still in progress [38].

\section{Comments and Conclusions}

We have presented a short review on the behavior of noninteracting brownian particles in the presence of periodic or random potentials. The wide range of phenomenologies includes dispersionless regimes, orthogonal velocity with respect the driving force, subtransport, superdiffusion, etc. Our interest is focused on determining whether such unexpected rich phenomenology is only controlled by simple mechanisms such as nonlinearities, thermal fluctuations, etc., and it is not necessary to include very specific assumptions such sophisticated external noises, fractional Fokker-Planck equations [39] or interactions between particles. In all the cases, the interplay between force, temperature and nonlinearities whithin the theoretical scenario of Langevin equations is sufficient to obtain these results. In any case, the presence of these unexpected regimes is quite remarkable given the simplicity of the model system.

Due to the finite observation time and system size, it is difficult to prove whether most of these regimes are transient or not. In some cases we have show that theses transients are larger than any other characteristic time of the system. In other cases we have tested that the phenomenology is stable using systems with different sizes. It is worth remarking that real experiments are performed in a finite time and size, so we expect that the predicted anomalies have to be observed. Particularly appealing is the exploration of the random disorder case, where more diversity of phenomenologies are expected if infradamped particles are used. To be more specific, we remark several examples in which different real systems may be studied by using the Langevin approach: controlled transport of paramagnetic colloidal particles above magnetic garnet films [40], charged particle transport in antidot lattices in the presence of magnetic and electric fields [41], and colloidal particles in landscapes generated by holographic optical traps [13]. Furthermore, the sorting mechanism can be used to focus a flow of particles [7]. This phenomenon has special relevance to mixing two beams of different particles in the laminar regime of the fluid, thus increasing the probability of an effective collision and reaction between them.

This paper is dedicated to our fiend Lutz Schimansky-Geier on the occasion of his 60th birthday. We aknowledge our collaborators Prof. K. Lindenberg, Prof. A. H. Romero and M. Khoury. We also 
acknowledge financial support from projects FIS20069-13360 (Ministerio de Educación y Ciencia of Spain) and 2009SGR14 (J.M.S.) and 2009SGR878 (A.M.L.) (Generalitat de Catalunya).

\section{References}

1. A. Einstein, Ann. Phys, 17, (1905) 549.

2. V. Blickle, T. Speck, C. Lutz, U. Seifert, C. Bechinger1, Phys. Rev. Lett. 98, (2007) 210601.

3. M. P. MacDonald, G. C. Spalding, K. Dholakia, Nature (London) 426, (2993) 421.

4. D. G. Grier, Nature (London) 424, (2003) 810.

5. P.T. Korda, M. B. Taylor, D. G. Grier, Phys. Rev. Lett. 89, (2002) 128301.

6. L. R. Huang, E. C. Cox, R. H. Austin, J. C. Sturm, Science 304, (2004) 987.

7. K. J. Morton, K. Loutherback, D.W. Inglis, O.K. Tsui, J.C. Sturm, S.Y. Chou, R.H. Austin, Proc. Natl. Acad. Sci. USA 105 (2008) 7434.

8. K. Lindenberg, J. M. Sancho, A. M. Lacasta, I. M. Sokolov, Phys. Rev. Lett. 98, (2007) 020602.

9. K. Lindenberg, A. M. Lacasta, J. M. Sancho, A. H. Romero, New Journal of Physics 7, (2005) 29.

10. P. Colet, M. San Miguel, J.M. Sancho, Phys. Rev. A 39 (1989) 149.

11. B. Lindner, M. Kostur, L. Schimansky-Geier, Fluct. Noise Lett. 1, (2001) R25.

12. P. Reimann, C. Van den Broeck, H. Linke, P. Hanggi, J. M. Rubi, A. Pérez-Madrid, Phys. Rev. Lett 87, (2001) 010602.

13. S. H. Lee, D. G. Grier, Phys. Rev. Lett. 96, (2006) 190601.

14. A. M. Lacasta, J. M. Sancho, A. H. Romero, K. Lindenberg, Phys. Rev. Lett. 94, (2005) 160601.

15. J. M. Sancho, M. Khoury, K. Lindenberg, A. M. Lacasta, J. Phys. Condensed Matter 17, (2005) S4151.

16. A. M. Lacasta, M. Khoury, J. M. Sancho, K. Lindenberg, Modern Physics Letters B 20, (2006) 1427.

17. J. P. Gleeson, J. M. Sancho, A. M. Lacasta, K. Lindenberg, Phys. Rev. E 73, (2006) 041102.

18. J.M. Sancho, A.M. Lacasta, K. Lindenberg, I.M. Sokolov, A.H. Romero, Phys. Rev. Lett. 92, (2004) 250601.

19. A.M. Lacasta, J.M. Sancho, A.H. Romero, I.M. Sokolov, K. Lindenberg, Phys. Rev. E70, (2004) 051104.

20. A.M. Lacasta, J.M. Sancho. Preprint (2010).

21. A. H. Romero, A. M. Lacasta, J. M. Sancho, Phys. Rev. E 69, (2004) 051105.

22. E. Heinsalu, M. Patriarca, F. Marchesoni, Phys. Rev. E 77, (2008) 021129.

23. D. Hennig, S. Martens, S. Fugmann, Phys. Rev.E 78, (2008) 011104.

24. M. Tiwari, S. Gonçalves, V. M. Kenkre, Eur. Phys. J-B 62, (2008) 459.

25. S. Gonçalves, C. Fusco, A. R. Bishop, V.M. Kenkre, Phys. Rev. E 72, (2005) 195418.

26. S. von Gehlen, M. Evstigneev, P. Reimann, Phys. Rev.E 77, (2008) 031136.

27. A. Taloni, F. Marchesoni Phys. Rev. Lett. 96, (2006) 020601.

28. H. Risken The Fokker-Planck Equation Springer-Verlag New York (1989).

29. C. Costantini, F. Marchesoni, Europhys. Lett. 48 (1999) 491.

30. J. G. Giddings, Unified Separation Science (John Wiley, New York, 1991).

31. C. Reichhardt, C. J. Olson Phys. Rev. Lett. 89 (2002) 078301.

32. P. Reimann, R. Eichhorn, Phys. Rev. Lett. 101, (2008) 180601.

33. J. García-Ojalvo, J.M. Sancho, Noise in Spatially Extended System (Springer, New York, 1999).

34. M. Khoury, J. O. Gleeson, J.M. Sancho, A. M. Lacasta, K. Lindenberg, Phys. Rev. E 80, (2009) 021123.

35. A. H. Romero, J. M. Sancho, Phys. Rev. E 58, (1998) 2833.

36. J-P. Bouchaud, A. Georges, Phys. Rep. 195, (1990) 127.

37. J. M. Sancho A. M. Lacasta, K. Lindenberg, I. M. Sokolov, A. H. Romero, Phys. Rev. Lett. 94, (2005) 188902.

38. M. Khoury, A.M. Lacasta, J.M. Sancho, K. Lindenberg, preprint (2010)

39. A. V. Chechkin, R. Gorenflo, I. M. Sokolov, Phys. Rev. E 66, (2002) 046129.

40. P. Tierno, F. Sagues, T. H. Johansen, T. M. Fisher, Phys. Chem. Chem. Phys. 11 (2009) 9615.

41. M. Khoury, A.M. Lacasta, J.M. Sancho, A.H. Romero, K. Lindenberg, Phys. Rev. B78, (2008) 155433. 